\title{
Expression of NOTCH1 in thyroid cancer is mostly restricted to papillary carcinoma
}

\author{
Simonetta Piana ${ }^{1}$, Eleonora Zanettii ${ }^{1}$ Alessandra Bisagni ${ }^{1}$, Alessia Ciarrocchi $^{2}$, Davide Giordano ${ }^{3}$, \\ Federica Torricelli ${ }^{2}$, Teresa Rossi ${ }^{2}$ and Moira Ragazzi ${ }^{1}$ \\ ${ }^{1}$ Pathology Unit, Azienda USL - IRCCS Reggio Emilia, Reggio Emilia, Italy \\ ${ }^{2}$ Laboratory of Translational Research, Azienda USL - IRCCS Reggio Emilia, Reggio Emilia, Italy \\ ${ }^{3}$ Otolaryngology Unit, Azienda USL - IRCCS Reggio Emilia, Reggio Emilia, Italy
}

Correspondence should be addressed to S Piana: Simonetta.Piana@ausl.re.it

\begin{abstract}
The NOTCH signaling is an evolutionarily conserved signaling pathway that regulates cell-cell interactions. NOTCH family members play a fundamental role in a variety of processes during development in particular in cell fate decisions. As other crucial factors during embryogenesis, NOTCH signaling is aberrantly reactivated in cancer where it has been linked to context-dependent effects. In thyroid cancer, NOTCH1 expression has been associated to aggressive features even if its in vivo expression within the entire spectrum of thyroid tumors has not definitively established. A series of 106 thyroid specimens including non-neoplastic lesions, benign and malignant tumors of common and rare histotypes, were investigated by immunohistochemistry to assess NOTCH1 expression. Extent of positivity and protein localization were investigated and correlated with clinical and morphological parameters. NOTCH1 positivity was predominantly associated with papillary carcinomas and only occasionally found in follicular carcinomas. Poorly differentiated and undifferentiated thyroid carcinomas showed only a partial positivity. NOTCH1 expression pattern also seemed differently distributed according to histotype. Our data confirm a role of NOTCH1 in thyroid cancer and highlight for the first time the specific involvement of this pathway in papillary carcinomas. Our data also indicate that other thyroid malignancies do not rely on NOTCH1 signaling for development and progression.
\end{abstract}

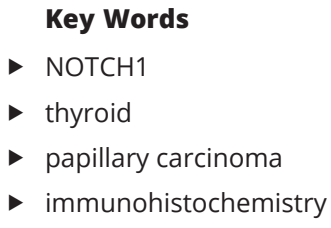

Endocrine Connections (2019) 8, 1089-1096

\section{Introduction}

NOTCH receptors belong to a highly conserved signaling pathway. Relying on cell-cell contacts, receptors belonging to this family sense cues from the surrounding microenvironment inducing intracellular responses.

$\mathrm{NOTCH}$ signaling regulates many fundamental processes during development and homeostasis of a wide range of tissues. As a consequence, deregulation of this pathway has been linked to multiple human disorders, from congenital syndromes to cancer $(1,2,3)$. NOTCH signaling is considered a potential target in several hematological malignancies (4) and carcinomas (5) and has a potential role in reducing tumor angiogenesis $(6,7)$.
Noticeably, integration of the large amount of genetic and functional data lately generated on the function of NOTCH signaling in cancer suggests a broad, often conflictual and context-dependent function $(8,9,10,11)$. Indeed, functional studies implicate NOTCH signaling as essential for many if not all hallmarks of cancer and clearly point to roles that range from oncogenic to tumor suppressive, depending on cancer type (12).

$\mathrm{NOTCH}$ receptors are large transmembrane proteins and $\mathrm{NOTCH}$ receptor activation is mediated by a sequence of proteolytic events, which cause the release of the NOTCH intracellular domain, translocation to

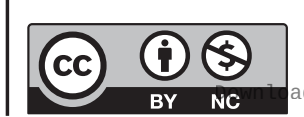


the nucleus and association with a DNA-bound protein (13). There are different expression patterns of the ligand and the receptors, explaining differences in signaling activity and post translational modifications, but there is a growing body of data demonstrating that $\mathrm{NOTCH}$ signaling is more complex than originally supposed and that its apparently contradictory role as oncogene or tumor suppressor has to be clarified yet (14).

NOTCH1 is the prototype and by far the most studied member of this family of receptors. NOTCH1 as other members of this family has been involved in the development of thyroid cancer even if with controversial role. On one side the activation of NOTCH signaling mediates growth suppression by cell cycle arrest in welldifferentiated thyroid carcinomas (WDTCs) $(15,16)$. On the other side, NOTCH1 has been described highly expressed in WDTC with BRAF, RET/papillary thyroid carcinoma (PTC) mutation or active MAPK signaling. In this context, activated NOTCH1 signaling promotes tumor growth $(17,18)$. Noticeably, Kim et al. postulated that NOTCH signaling influences the malignant potential of thyroid cancer and observed a NOTCH1-dependent reduction of epithelial-mesenchymal transition (EMT) and cancer stem cells markers in anaplastic tumors (19). In parallel, the expression of NOTCH1 receptor seems to be related to tumor invasiveness in PTC, and it has been proposed as molecular marker of poor prognosis (20, $21)$. In line with this reports, we recently reported that NOTCH1 cooperates with the transcription factor DEC1 in controlling aggressiveness of PTCs (22).

Despite the involvement of NOTCH1 in thyroid carcinogenesis, still many issues remain to be addressed. In particular, the expression profile of this protein within the spectrum of thyroid tumors, benign and malignant, follicular-derived and medullary, has never been consistently investigated (23). Thus, in this work, we aimed at analyzing by immunohistochemistry the expression of NOTCH1 in a large series of thyroid neoplasms with distinct histogenesis and very different biological behavior.

\section{Materials and methods}

\section{Case selection}

The study was conducted by the Pathology Unit of the Arcispedale Santa Maria Nuova-IRCCS in Reggio Emilia, Italy, and was approved by the Local Ethics Committee (namely, Comitato Etico di Area Vasta Emilia Nord, protocol Rf-2016-02364167, approved 29th June 2017) that authorized the use of anonymized archive tissue. One hundred six surgically excised thyroid samples were selected from the archive of the Pathology Unit and re-evaluated according to the current 2017 WHO classification (24). Clinicopathological information are summarized in Table 1 and Supplementary Table 1 (see section on supplementary data given at the end of this article).

\section{Immunostaining}

Immunohistochemistry was performed on a representative $4 \mu \mathrm{m}$ formalin-fixed, paraffin-embedded section from each case, as previously described (25), including both neoplastic and normal thyroid tissues.

Rabbit polyclonal NOTCH1 antibody (Cell Signaling, clone D1E11, code \#3608) and monoclonal Ki67

Table 1 Clinical and pathologic features of the patients $(N=106)$

\begin{tabular}{lcc}
\hline & $\boldsymbol{N}(\%)$ \\
\cline { 1 - 1 } Age at diagnosis, years (mean, 95\% Cl) & $54(19-85)$ \\
Fender & & \\
Males & & \\
Histological diagnosis & & $(63.2)$ \\
NH & & \\
FA & $2(1.9)$ \\
HA & $5(4.7)$ \\
HTT & $5(4.7)$ \\
PTC & $6(5.7)$ \\
FTC & $48(45.3)$ \\
HCC & $12(11.3)$ \\
MTC & $5(4.7)$ \\
PDTC & $9(8.5)$ \\
ATC & $6(5.7)$ \\
Stage (AJCC) & $8(7.5)$ \\
NA & \\
I & $19(17.9)$ \\
II & $61(57.5)$ \\
III & $12(11.3)$ \\
IV & $0(0)$ \\
Metastases (lymph node + distant) & $14(13.3)$ \\
Yes & \\
No & $28(26.4)$ \\
NA & $60(56.6)$ \\
Number of metastases & $18(17)$ \\
Single & \\
Multiple & $13(12.3)$ \\
NA & $13(12.3)$ \\
\hline
\end{tabular}

ATC, anaplastic thyroid carcinoma; FA, follicular adenoma; FTC, follicular thyroid carcinoma; HA, Hürthle cell adenoma; HCC, Hürthle cell carcinoma; HTT, hyalinizing trabecular tumor; MTC, medullary thyroid carcinoma; NA, not available; $\mathrm{NH}$, nodular hyperplasia; PDTC, poorly differentiated thyroid carcinoma; PTC, papillary thyroid carcinoma.

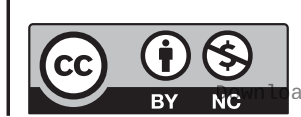


antibody (clone MIB1, Agilent) were used at $0.33 \mu \mathrm{g} / \mathrm{mL}$ and $46 \mathrm{mg} / \mathrm{mL}$ respectively, according to manufacturer's instructions. In a previous work we confirmed the NOTCH1 antibody specificity by performing Western blot analysis on thyroid cancer cell lines transfected with siRNA against NOTCH1 (22).

As positive controls, we stained samples from human colic mucosa and other epithelia, in accordance with the indications of the antibody distributors. As negative control, we used the normal thyroid tissue adjacent to the lesions examined, which was consistently negative in all cases. To further verify the specificity of the antibody, according to the Tumor Protein Atlas (https:// www.proteinatlas.org/ENSG00000148400-NOTCH1/ tissue/ovary), we additionally performed NOTCH1 immunostaining on three cases of normal ovarian tissue, three cases of peripheral nerves in traumatic neuroma, three cases of hyperplastic lymphoid tissue of the tonsil and three cases of normal pancreatic tissue, including both exocrine pancreas and islets of Langerhans. As expected, we did not observe any staining in the ovarian stroma (Supplementary Fig. 1A), peripheral nerve (Supplementary Fig. 1B), lymphoid germinal centers (while squamous epithelium was reactive, Supplementary Fig. 1C) and islets of Langerhans (on a background of positive exocrine pancreas, Supplementary Fig. 1D), thus confirming the specificity of the staining.

The tissue sections were pre-treated with heat antigen retrieval in EDTA buffer for $72^{\prime}$. The staining was developed on a Ventana Bench-Mark ULTRA platform using the OptiView DAB detection Kit (Ventana-Roche, Tucson, USA). Slides were counterstained with hematoxylin.

The entire section from each case was scored for signals quantification by two pathologists (SP and MR). The signal was defined as absent (neg, 0\%), focal $(+, 1-29 \%)$, moderate $(++, 30-50 \%)$ and diffuse $(+++,>50 \%)$.
Pattern of positivity was recorded as luminal (i.e. on the apical pole of the neoplastic cells), membranous and cytoplasmatic.

\section{Statistical analysis}

Statistical analysis was performed using R Software v 3.5.1. Analysis of the frequency of NOTCH1 positivity was performed by contingency tables and significance was calculated by Fisher's exact test. Differences were considered statistically significant with a $P$ value $<0.05$.

\section{Results}

\section{Patients' features}

One hundred six thyroid tumors, spanning the entire spectrum of thyroid lesions were analyzed (Table 1 and Supplementary Table 1). Sixty-three percent of patients were female (67 out of 106) and the average age was $54 \pm 15$ years. Two samples were nodular hyperplasia $(\mathrm{NH})$, five samples were follicular adenomas (FA), five were Hürthle cell adenoma (HA) and six were hyalinizing trabecular tumors (HTT). The remaining were malignant lesions. $68.1 \%$ were classical well-differentiated thyroid cancers, papillary thyroid carcinoma (PTC, $n=48,54.5 \%$ ) and follicular thyroid carcinoma (FTC, $n=12,13.6 \%$ ). $15.9 \%$ were scarcely differentiated tumors, including poorly differentiated thyroid carcinoma (PDTC, $n=6$, $6.8 \%$ ) and anaplastic thyroid carcinoma (ATC, $n=8$, 9.1\%). The remaining $31.9 \%$ of samples encompassed rare histological variants of thyroid cancer including Hürthle cell carcinomas (HCC, $n=5,5.7 \%$ ) and medullary thyroid carcinoma (MTC, $n=9,10.2 \%$ ). Twenty-eight malignant tumors had developed metastasis (26.4\%) and 13 of these had multiple metastatic lesions (46.4\%).

Table 2 Results of NOTCH1 expression.

\begin{tabular}{l}
\hline NOTCH1 immunostaining \\
\hline Negative \\
+ \\
++ \\
+++ \\
TOT \\
\hline NOTCH1 pattern \\
\hline Cytoplasmic \\
Luminal \\
Cytoplasmic + luminal \\
TOT-positive cases
\end{tabular}

\begin{tabular}{c}
\hline Non-neoplastic/benign tumors (\%) \\
\hline $15(83.2)$ \\
$1(5.6)$ \\
$1(5.6)$ \\
$1(5.6)$ \\
$18(100)$ \\
\hline Non-neoplastic/benign tumors (\%) \\
\hline $3(100)$ \\
0 \\
$3(100)$
\end{tabular}

\begin{tabular}{c}
\hline Malignant tumors $(\%)$ \\
\hline $28(31.8)$ \\
$14(15.9)$ \\
$3(3.4)$ \\
$43(48.9)$ \\
$88(100)$ \\
\hline Malignant tumors $(\%)$ \\
\hline $11(18.3)$ \\
$48(80)$ \\
$1(1.7)$ \\
$60(100)$
\end{tabular}

\begin{tabular}{c}
\hline $\boldsymbol{N}(\%)$ \\
\hline $43(40.6)$ \\
$15(14.2)$ \\
$4(3.7)$ \\
$44(41.5)$ \\
$106(100)$ \\
\hline $\boldsymbol{N}(\%)$ \\
\hline $14(22.2)$ \\
$48(76.2)$ \\
$1(1.6)$ \\
$63(100)$
\end{tabular}


Metastases were associated primarily with ATC $(n=7)$, PTC $(n=9)$ and MTC $(n=3)$.

\section{Immunohistochemical expression of NOTCH1}

Tables 2 and 3 summarize the results of this analysis. Both positivity and pattern of expression were evaluated. NOTCH1 expression was significantly associated with malignant phenotype $(P<0.0011)$. Sixty-eight percent of malignant lesions (60 out of 88; 45 PTC, 5 FTC, 4 PDTC, 3 ATC, 2 MTC and 1 HCC) were positive for NOTCH1, while only $16.6 \%$ of positive non-malignant samples (3 out 18) scored positive for this marker. NOTCH1 positivity in the non-malignant lesions were restricted to FA. $69.8 \%$ of positive lesions showed NOTCH1 expression in more than $50 \%$ of cancer cells (Supplementary Table 2).

Distribution of NOTCH1 positivity per histotype is reported in Fig. 1 and Table 3. NOTCH1 positivity was significantly associated with PTCs. $93.75 \%$ of PTCs tested were positive for NOTCH1 and $82.2 \%$ of these (37 out of 48 ) showed NOTCH1 positivity in over $50 \%$ of cancer cells (Fig. 1). NOTCH1 expression was also observed in $41.6 \%$ of FTCs (5 out 12), $66.7 \%$ of PDTC (4 out of 6 ) and $37.5 \%$ of ATC (3 out of 8 ). By contrast NOTCH1 was limitedly expressed in MTC (two out of nine) and

Table 3 NOTCH1 positivity according to histotype.

\begin{tabular}{l}
\hline Positivity (TOT) \\
\hline NH (2) \\
FA (5) \\
HA (5) \\
HTT (6) \\
PTC (48) \\
FTC (12) \\
HCC (5) \\
MTC (9) \\
PDTC (6) \\
ATC (8)
\end{tabular}

\section{Pattern (TOT-NOTCH1+)}

FA (3)

PTC (45)

FTC (5)

$\mathrm{HCC}(1)$

MTC (2)

PDTC (4)

ATC (3)

\begin{tabular}{c}
\hline Pos $(\%)$ \\
\hline $0(0)$ \\
$3(60)$ \\
$0(0)$ \\
$0(0)$ \\
$45(93.75)$ \\
$5(41.6)$ \\
$1(20)$ \\
$2(22.2)$ \\
$4(66.7)$ \\
$3(37.5)$ \\
\hline Cytoplasmic $(\%)$ \\
\hline $3(100)$ \\
$0(0)$ \\
$3(60)$ \\
$1(100)$ \\
$2(100)$ \\
$3(75)$ \\
3* (100)
\end{tabular}

\begin{tabular}{c}
\hline Neg $(\%)$ \\
\hline $2(100)$ \\
$2(40)$ \\
$5(100)$ \\
$6(100)$ \\
$3(6.25)$ \\
$7(58.3)$ \\
$4(80)$ \\
$7(77.8)$ \\
$2(33.3)$ \\
$5(62.5)$ \\
\hline Luminal $(\%)$ \\
\hline $0(0)$ \\
$45(100)$ \\
$2(40)$ \\
$0(0)$ \\
$0(0)$ \\
$1(25)$ \\
$0(0)$
\end{tabular}

*One case of ATC shows both cytoplasmic and luminal positivity. ATC, anaplastic thyroid carcinoma; FA, follicular adenoma; FTC, follicular thyroid carcinoma; HA, Hürthle cell adenoma; HCC, Hürthle cell carcinoma; HTT, hyalinizing trabecular tumor; MTC, medullary thyroid carcinoma; $\mathrm{NH}$, nodular hyperplasia; PDTC, poorly differentiated thyroid carcinoma; PTC, papillary thyroid carcinoma.

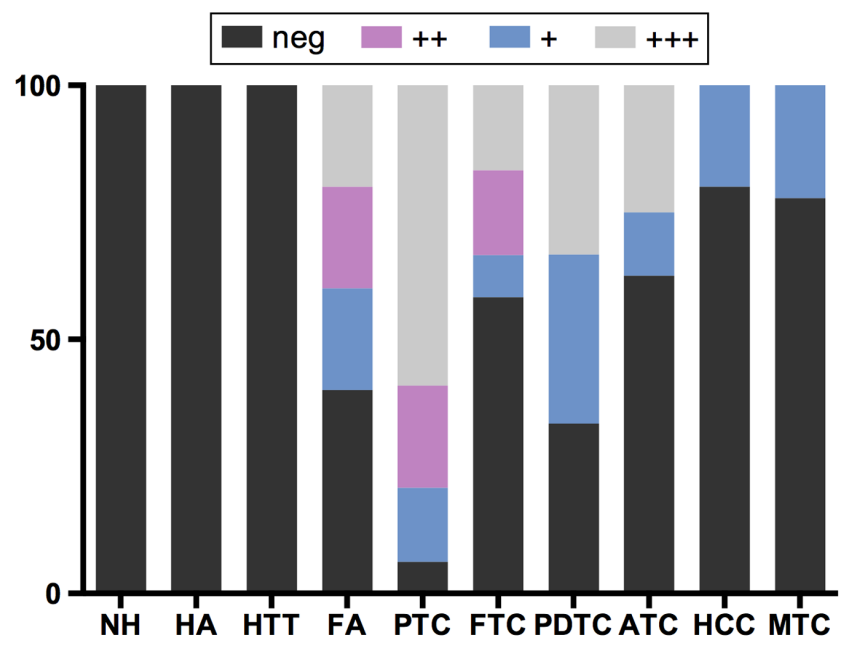

Figure 1

Distribution of NOTCH1 positivity in thyroid lesions. Histograms represent the percentage of negative and positive cells according to histotypes $(+=1-29 \%,++=30-50 \%,+++>50 \%)$. ATC, anaplastic thyroid carcinoma; FA, follicular adenoma; FTC, follicular thyroid carcinoma; HA, Hürthle cell adenoma; HCC, Hürthle cell carcinoma; HTT, hyalinizing trabecular tumor; MTC, medullary thyroid carcinoma; NH, nodular hyperplasia; PDTC, poorly differentiated thyroid carcinoma; PTC, papillary thyroid carcinoma.

HCC (one out of five) and in less than $10 \%$ of cells. As all the analyzed samples were surgical specimens, normal thyroid tissue was present in all cases and was consistently negative for NOTCH1 (Fig. 2).

Next, we focus our attention on localization of NOTCH1 positivity (Table 3 ). Strikingly, all positive PTCs showed luminal localization of NOTCH1 regardless of the sub-histotype (classic, tall cell, follicular) as the staining was stronger in the cell surface toward the lumen of the follicles or the papillae (Fig. 3). Luminal localization of NOTCH1 was largely associated to PTCs, while in the other histotype NOTCH1 was detected primarily in the cytoplasm.

Among the NOTCH1-positive samples, three PDTCs (75\%) showed a granular cytoplasmic positivity (Fig. 4). In one PDTC, cytoplasmic granularity was thick and dot-like (Fig. 4D). Both NOTCH1-positive MTCs showed cytoplasmic positivity even if the staining was focal and limited to few neoplastic elements (Fig. A and B). Finally, positive FA, FTC and AC showed a delicate granular positivity in the cytoplasm (Fig. 5).

To explore whether high NOTCH1 expression could reflect higher proliferation rate of these tumors, we explored Ki67 expression in the 48 PTCs included in this analysis. In all samples, Ki67 positivity was limited (below $10 \%$ of cancer cells) independently to NOTCH1 positivity. This is not surprising, since it was already reported that Ki67 levels in WDTCs are low (26).

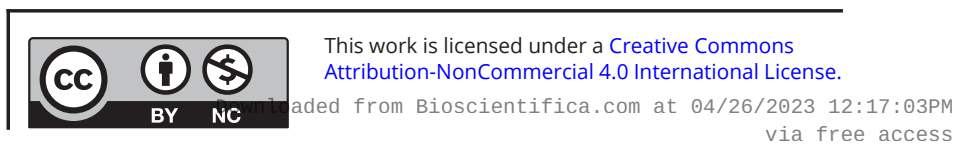




\begin{tabular}{|c|c|c|c|c|}
\hline Endocrine & S Piana et al. & $\begin{array}{l}\text { NOTCH1 expression in thyroid } \\
\text { tumors }\end{array}$ & $8: 8$ & 1093 \\
\hline
\end{tabular}
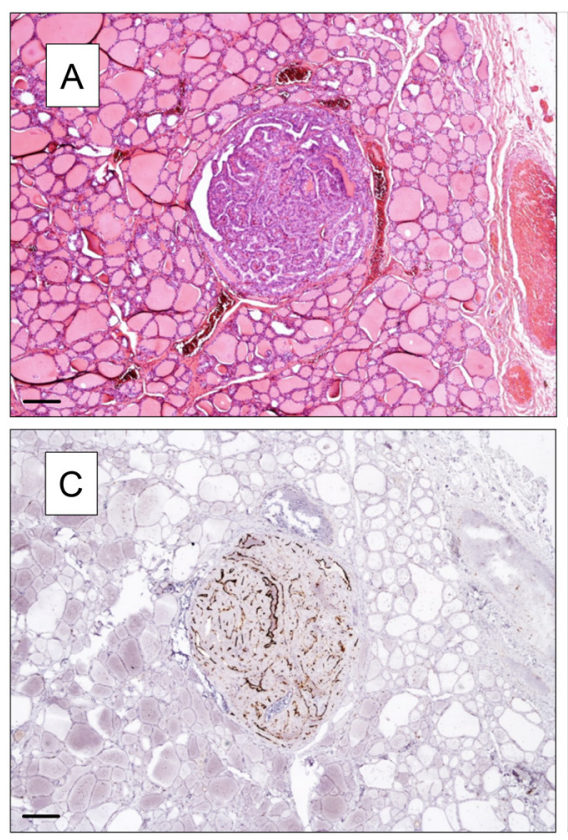

\section{Figure 2}

NOTCH1 expression in a papillary microcarcinoma and in the normal surrounding thyroid tissue. (A and B) H\&E staining (in B microcarcinoma at higher power view). (C and D) NOTCH1 staining. NOTCH1 positivity is restricted to microcarcinoma (in the center; in $\mathrm{D}$ at higher power view), while normal thyroid tissue is negative. (A, B and $C$ ) Magnification 40×, (B, C and D) 100×. Scale bar $100 \mu \mathrm{m}$.
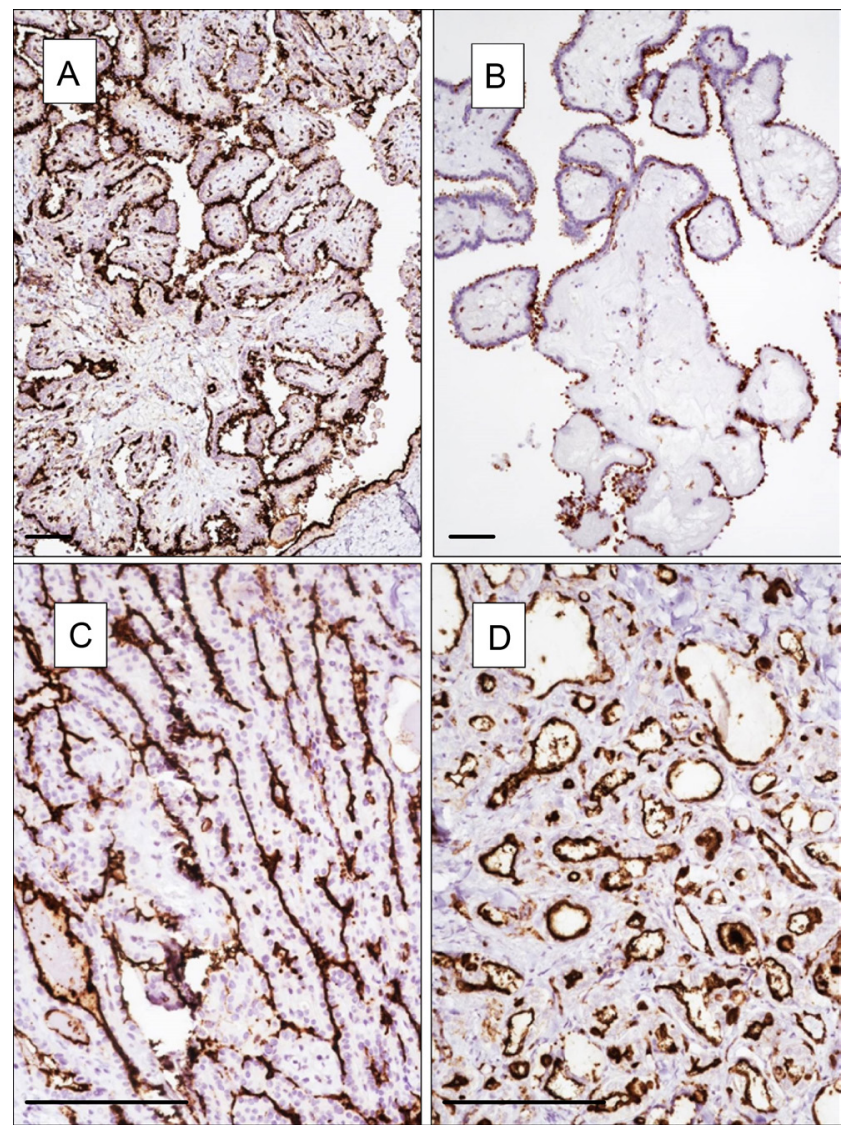

Figure 3

Pattern of NOTCH1 positivity in PTC classic type (A and B), tall cell variant (C) and follicular variant (D). (A and B) Magnification 40x, (C and D) 100x. Scale bar $100 \mu \mathrm{m}$.

https://ec.bioscientifica.com https://doi.org/10.1530/EC-19-0303

() 2019 The authors Published by Bioscientifica Ltd

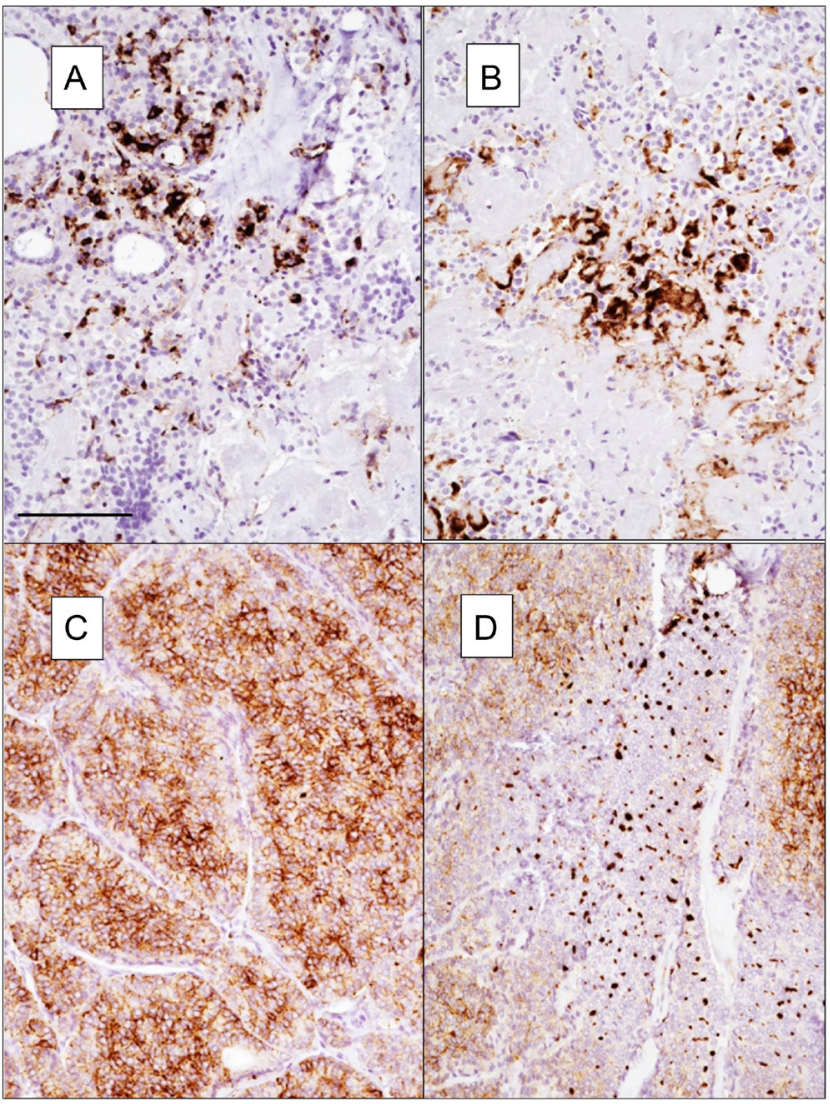

Figure 4

Pattern of NOTCH1 expression in MTC (A and B) and PDTC (C and D). In PDTC cytoplasmic granularity is thick and dot-like. Magnification 100x. Scale bar $100 \mu \mathrm{m}$.

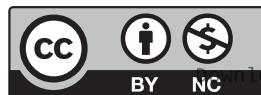

This work is licensed under a Creative Commons Attribution-NonCommercial 4.0 International License. ded from Bioscientifica.com at $04 / 26 / 202$ 


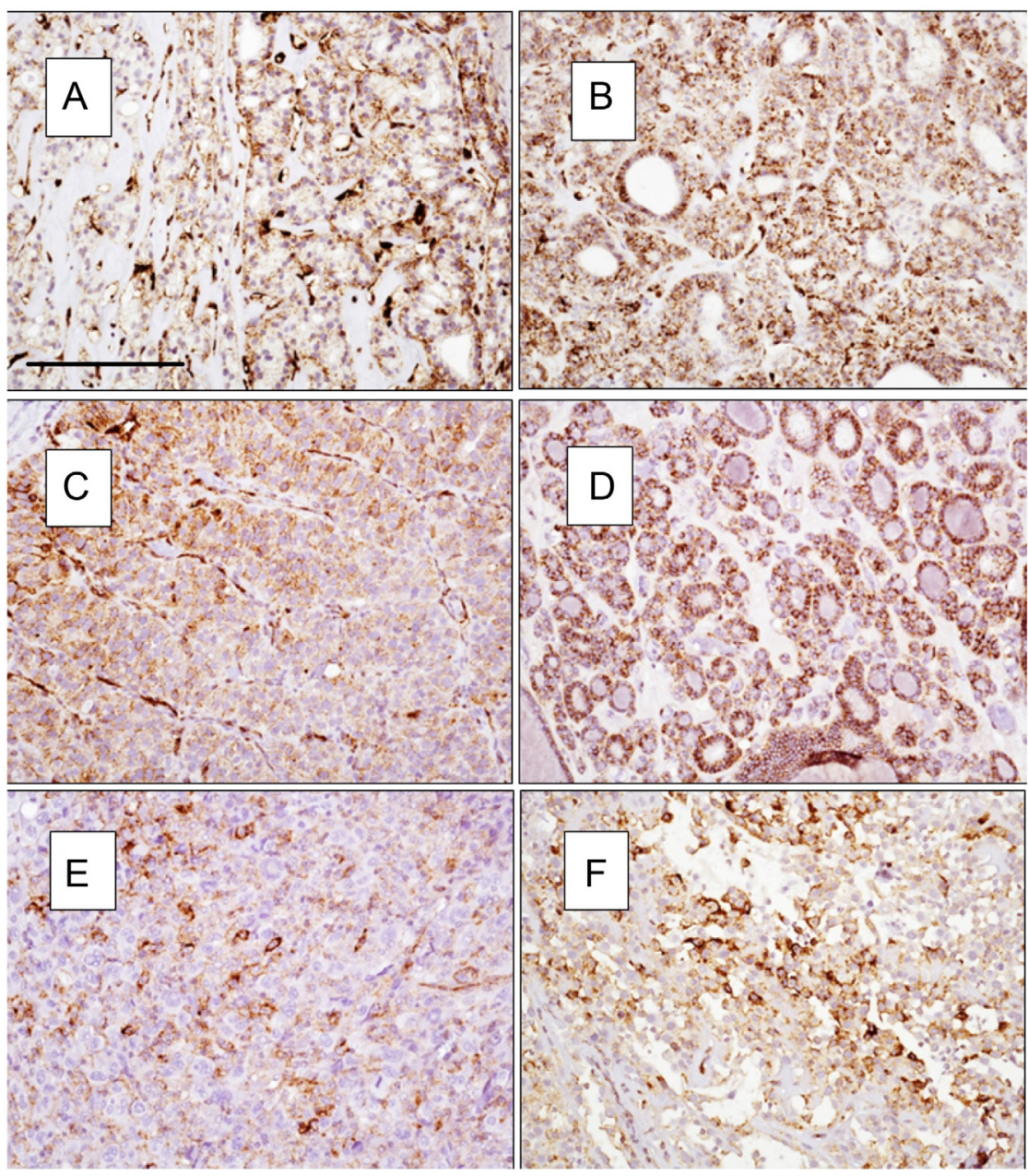

\section{Figure 5}

Pattern of NOTCH1 positivity in FA (A and B), FTC (C and D) and ATC (E and F). Magnification 100x. Scale bar $100 \mu \mathrm{m}$

\section{Discussion}

NOTCH signaling is an evolutionarily conserved intercellular pathway regulating interactions between adjacent cells. NOTCH1 is one of the four known members of this family. As the others, NOTCH1 protein is characterized by N-terminal EGF-like repeats followed by a Lin-12/NOTCH repeat (LNR) domains, specific of this family which prevents signaling activation. Once engaged by its ligand, NOTCH1 exposes a site of cleavage that allows ADAM17, and then $\gamma$-secretase cleavage that releases in the cytoplasm NICD, the activated form of NOTCH1. NICD is then translocated to the nucleus where, by the cooperation with transcriptional co-factors, controls gene expression (13). NOTCH1 signaling plays a crucial role during embryonic development being involved in cell fate commitment and differentiation (27). In cancer, NOTCH1 has been shown to play multiple and often controversial functions (28). This is evident also in the setting of thyroid cancer where, in spite of multiple reports, a definitive consensus on NOTCH1 involvement is still missing.

Yu and colleagues have shown that NOTCH1 mRNA levels decrease in thyroid tumor cells as compared to normal thyroid and that patients with lower NOTCH1 expression had a significantly higher recurrence rate $(P=0.038)(21)$. By contrast, Kim et al. reported that the expression of activated NOTCH1 was higher in ATCs than in PTCs indicating a role of this pathway in aggressive evolution of thyroid cancer (19). According to these authors, inhibition of NOTCH1 significantly reduced proliferation and migration of ATC cells, but not PTC cells. In contrast with this observation several reports indicate a loss of NOTCH1 expression in ATCs $(15,29$, 30 ), and we recently showed that NOTCH1 participates to the acquisition of aggressive features in PTC cells (22). Beside these controversies, no information on NOTCH1 expression was available in rare histological subtypes. Here, we showed that (1) NOTCH1 expression is restricted to tumor cells and significantly associated with malignant https://ec.bioscientifica.com https://doi.org/10.1530/EC-19-0303

C) 2019 The authors Published by Bioscientifica Ltd
This work is licensed under a Creative Commons Attribution-NonCommercial 4.0 International License. ded from Bioscientifica.com at 04/26/2023 12:17:03PM 
phenotype in follicular-derived tumors since only 3 of the 18 benign lesions showed expression of this protein; (2) NOTCH1 expression is largely associated with PTC, while its expression in poorly differentiated tumors is less consistent; (3) rare variants including the C-cellsderived medullary carcinomas do not express NOTCH1 or have very low NOTCH1 levels and likely do not rely on this signaling for their progression. Due to the high rate of positivity observed in the PTC group, we did not notice significant association with clinical-pathologic features of these tumors. In this regard our data are in partial contradiction with the meta-analysis reported by Yuan et al. that reported a positive association of NOTCH1 expression in PTCs with lymph node metastasis, tumor size, capsular invasion and clinical stage (31).

Being NOTCH1 proteolitically processed and translocated into the nucleus upon activation, analysis of its localization in human tumors has been used to predict NOTCH1 pathway activation (32). In this regard our data indicate that in PTCs in which NOTCH1 is consistently and widely expressed, this protein is retained in the membrane. Noticeably NOTCH1 expression does not mark the entire membrane surface of thyrocytes, but it is restricted to the luminal surface of the papillae, even when their structure is partially compromised by the irregular neoplastic growth. Luminal expression of NOTCH1 is instead lost in poorly differentiated and undifferentiated lesions, likely as reflex of the cellular morphological changes imposed by the 'undifferentiation' process. In our analysis, we did not observe nuclear localization of NOTCH1, and in most of the non-PTC tumors, this protein seems to be localized in the cytoplasm with a granular pattern. We do not have an explanation regarding the biological meaning of this localization, but it is possible that it reflects retention of this protein in the reticulum, where it may be processed for degradation. If, as it is plausible, NOTCH1 cytoplasmic localization underscore a non-functional protein, thus our data indicate that NOTCH1 signaling, in the setting of thyroid carcinomas, operates exclusively in the PTC variants.

Even if further analyses are needed, our data add information on the potential function of NOTCH1 signaling on thyroid cancers and point to a specific role of this pathway on well-differentiated and most common variants of this tumors.

\section{Supplementary data}

This is linked to the online version of the paper at https://doi.org/10.1530/ EC-19-0303.

\section{Declaration of interest}

The authors declare that there is no conflict of interest that could be perceived as prejudicing the impartiality of the research reported.

\section{Funding}

This research was funded by Italian Ministry of Health, grant number RF-2016-02364167.

\section{References}

1 Lobry C, Oh P \& Aifantis I. Oncogenic and tumor suppressor functions of NOTCH in cancer: it's NOTCH what you think. Journal of Experimental Medicine 2011208 1931-1935. (https://doi. org/10.1084/jem.20111855)

2 Louvi A \& Artavanis-Tsakonas S. NOTCH and disease: a growing field. Seminars in Cell and Developmental Biology 201223 473-480. (https://doi.org/10.1016/j.semcdb.2012.02.005)

3 Nowell CS \& Radtke F. NOTCH as a tumour suppressor. Nature Reviews: Cancer 201717 145-159. (https://doi.org/10.1038/ nrc.2016.145)

$4 \mathrm{Gu}$ YS, Masiero M \& Banham AH. NOTCH signaling: its roles and therapeutic potential in hematological malignancies. Oncotarget 2016 7 29804-29823. (https://doi.org/10.18632/oncotarget.7772)

5 Venkatesh V, Nataraj R, Thangaraj GS, Karthikeyan M, Gnanasekaran A, Kaginelli SB, Kuppanna G, Kallappa CG \& Basalingappa KM. Targeting NOTCH signaling pathway of cancer stem cells. Stem Cell Investigation 20185 5. (https://doi.org/10.21037/ sci.2018.02.02)

6 Dufraine J, Funahashi Y \& Kitajewski J. NOTCH signaling regulates tumor angiogenesis by diverse mechanisms. Oncogene 200827 5132-5137. (https://doi.org/10.1038/onc.2008.227)

7 Kofler NM, Shawber CJ, Kangsamaksin T, Reed HO, Galatioto J \& Kitajewski J. NOTCH signaling in developmental and tumor angiogenesis. Genes and Cancer 20112 1106-1116. (https://doi. org/10.1177/1947601911423030)

8 Brzozowa-Zasada M, Piecuch A, Dittfeld A, Mielanczyk L, Michalski M, Wyrobiec G, Harabin-Slowinska M, Kurek J \& Wojnicz R. NOTCH signaling pathway as an oncogenic factor involved in cancer development. Contemporary Oncology 201620 267-272. (https://doi.org/10.5114/wo.2016.61845)

9 Qi RZ, An HZ, Yu YZ, Zhang MH, Liu SX, Xu HM, Guo ZH, Cheng T \& Cao XT. NOTCH1 signaling inhibits growth of human hepatocellular carcinoma through induction of cell cycle arrest and apoptosis. Cancer Research 200363 8323-8329.

10 Wang Z, Da Silva TG, Jin K, Han X, Ranganathan P, Zhu X, SanchezMejias A, Bai F, Li B, Fei DL, et al. NOTCH signaling drives stemness and tumorigenicity of esophageal adenocarcinoma. Cancer Research 201474 6364-6374. (https://doi.org/10.1158/0008-5472.CAN-142051)

11 Zage PE, Nolo R, Fang W, Stewart J, Garcia-Manero G \& ZweidlerMcKay PA. NOTCH pathway activation induces neuroblastoma tumor cell growth arrest. Pediatric Blood and Cancer 201258 682-689. (https://doi.org/10.1002/pbc.23202)

12 Aster JC, Pear WS \& Blacklow SC. The varied roles of NOTCH in cancer. Annual Review of Pathology 201712 245-275. (https://doi. org/10.1146/annurev-pathol-052016-100127)

13 Borggrefe T \& Oswald F. The NOTCH signaling pathway: transcriptional regulation at NOTCH target genes. Cellular and Molecular Life Sciences 200966 1631-1646. (https://doi.org/10.1007/ s00018-009-8668-7)

14 Radtke F \& Raj K. The role of NOTCH in tumorigenesis: oncogene or tumour suppressor? Nature Reviews Cancer 20033 756-767. (https:// doi.org/10.1038/nrc1186)

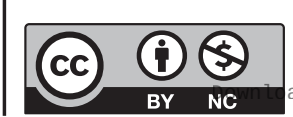

This work is licensed under a Creative Commons Attribution-NonCommercial 4.0 International License. ded from Bioscientifica.com at 04/26/2023 12:17:03PM 
15 Ferretti E, Tosi E, Po A, Scipioni A, Morisi R, Espinola MS, Russo D, Durante C, Schlumberger M, Screpanti I, et al. NOTCH signaling is involved in expression of thyrocyte differentiation markers and is down-regulated in thyroid tumors. Journal of Clinical Endocrinology and Metabolism 200893 4080-4087. (https://doi.org/10.1210/ jc.2008-0528)

16 Xiao X, Ning L \& Chen H. NOTCH1 mediates growth suppression of papillary and follicular thyroid cancer cells by histone deacetylase inhibitors. Molecular Cancer Therapeutics 20098 350-356. (https:// doi.org/10.1158/1535-7163.MCT-08-0585)

17 Yamashita AS, Geraldo MV, Fuziwara CS, Kulcsar MA, Friguglietti CU, da Costa RB, Baia GS \& Kimura ET. NOTCH pathway is activated by MAPK signaling and influences papillary thyroid cancer proliferation. Translational Oncology 20136 197-205. (https://doi. org/10.1593/tlo.12442)

18 Zhang MD, Qin YY, Zuo B, Gong W, Zhang SL, Gong YR, Quan ZW \& Chu BF . Overexpression of NOTCH-regulated ankyrin repeat protein is associated with papillary thyroid carcinoma progression. PLoS ONE 201712 e0167782. (https://doi.org/10.1371/journal.pone.0167782)

19 Kim HJ, Kim MJ, Kim A, Jung CW, Park S, Koh JS \& Myung JK. The role of NOTCH1 signaling in anaplastic thyroid carcinoma. Cancer Research and Treatment 201749 509-517. (https://doi.org/10.4143/ crt.2016.214)

$20 \mathrm{Fu}$ HL, Ma C, Guan WB, Cheng WW, Feng F \& Wang H. Expression of NOTCH 1 receptor associated with tumor aggressiveness in papillary thyroid carcinoma. OncoTargets and Therapy 20169 1519-1523. (https://doi.org/10.2147/OTT.S98239)

21 Yu XM, Jaskula-Sztul R, Georgen MR, Aburjania Z, Somnay YR, Leverson G, Sippel RS, Lloyd RV, Johnson BP \& Chen H. NOTCH1 signaling regulates the aggressiveness of differentiated thyroid cancer and inhibits SERPINE1 expression. Clinical Cancer Research 201622 3582-3592. (https://doi.org/10.1158/1078-0432.CCR-15-1749)

22 Gallo C, Fragliasso V, Donati B, Torricelli F, Tameni A, Piana S \& Ciarrocchi A. The bHLH transcription factor DEC1 promotes thyroid cancer aggressiveness by the interplay with NOTCH1. Cell Death and Disease 20189 871. (https://doi.org/10.1038/s41419-018-0933-y)

23 Geers C, Colin IM \& Gerard AC. Delta-like 4/NOTCH pathway is differentially regulated in benign and malignant thyroid tissues. Thyroid 201121 1323-1330. (https://doi.org/10.1089/thy.2010.0444)
24 Lloyd RV, Osmura RY, Klöppel G \& Rosai J.Tumors of the thyroid gland. In WHO Classification of Tumors of Endocrine Organs, pp 65-142. Lyon, France: IARC Press, 2017.

25 Sancisi V, Borettini G, Maramotti S, Ragazzi M, Tamagnini I, Nicoli D, Piana S \& Ciarrocchi A. Runx2 isoform I controls a panel of proinvasive genes driving aggressiveness of papillary thyroid carcinomas. Journal of Clinical Endocrinology and Metabolism 201297 E2006-E2015. (https://doi.org/10.1210/jc.2012-1903)

26 Tallini G, Garcia-Rostan G, Herrero A, Zelterman D, Viale G, Bosari S \& Carcangiu ML. Downregulation of p27Kip1 and Ki67/ Mib1 labeling index support the classification of thyroid carcinoma into prognostically relevant categories. American Journal of Surgical Pathology 199923 678-685. (https://doi.org/10.1097/00000478199906000-00007)

27 Hansson EM, Lendahl U \& Chapman G. NOTCH signaling in development and disease. Seminars in Cancer Biology 200414 320-328. (https://doi.org/10.1016/j.semcancer.2004.04.011)

28 Ayaz F \& Osborne BA. Non-canonical NOTCH signaling in cancer and immunity. Frontiers in Oncology 20144 345. (https://doi. org/10.3389/fonc.2014.00345)

29 Patel PN, Yu XM, Jaskula-Sztul R \& Chen H. Hesperetin activates the NOTCH1 signaling cascade, causes apoptosis, and induces cellular differentiation in anaplastic thyroid cancer. Annals of Surgical Oncology 201421 S497-S504. (https://doi.org/10.1245/s10434-013-3459-7)

30 Yuan L, Ma L, Xue H \& Song S. Relationship between the upregulation of Notch1 signaling and the clinical characteristics of patients with papillary thyroid carcinoma in East Asia: a systematic review and meta-analysis. Cancer Cell International 2019195. (https://doi.org/10.1186/s12935-018-0723-8)

31 Yu XM, Jaskula-Sztul R, Ahmed K, Harrison AD, Kunnimalaiyaan M \& Chen H. Resveratrol induces differentiation markers expression in anaplastic thyroid carcinoma via activation of NOTCH1 signaling and suppresses cell growth. Molecular Cancer Therapeutics 201312 1276-1287. (https://doi.org/10.1158/1535-7163.MCT-12-0841)

32 Saito S, Ishiguro H, Kimura M, Ogawa R, Miyai H, Tanaka T, Mizoguchi K \& Takeyama H. Clinical significance of NOTCH1 intracellular cytoplasmic domain translocation into the nucleus in gastric cancer. Biomedical Reports 20165 344-348. (https://doi. org/10.3892/br.2016.723)

Received in final form 26 June 2019

Accepted 2 July 2019

Accepted Preprint published online 2 July 2019 https://ec.bioscientifica.com https://doi.org/10.1530/EC-19-0303 (c) 2019 The authors Published by Bioscientifica Ltd

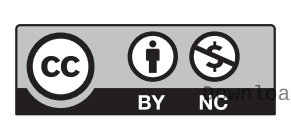

This work is licensed under a Creative Commons Attribution-NonCommercial 4.0 International License. ded from Bioscientifica.com at $04 / 26 / 2023$ 12:17:03PM 